\title{
Permanent magnet thrust bearings for flywheel energy storage systems: Analytical, numerical, and experimental comparisons
}

Dagnæs-Hansen, Nikolaj Aleksander; Santos, IImar F.

Published in:

Proceedings of the Institution of Mechanical Engineers, Part C: Journal of Mechanical Engineering Science

Link to article, DOI:

$10.1177 / 0954406219843952$

Publication date:

2019

Document Version

Peer reviewed version

Link back to DTU Orbit

Citation (APA):

Dagnæs-Hansen, N. A., \& Santos, I. F. (2019). Permanent magnet thrust bearings for flywheel energy storage systems: Analytical, numerical, and experimental comparisons. Proceedings of the Institution of Mechanical Engineers, Part C: Journal of Mechanical Engineering Science, 233(15), 5280-5293. https://doi.org/10.1177/0954406219843952

\section{General rights}

Copyright and moral rights for the publications made accessible in the public portal are retained by the authors and/or other copyright owners and it is a condition of accessing publications that users recognise and abide by the legal requirements associated with these rights.

- Users may download and print one copy of any publication from the public portal for the purpose of private study or research.

- You may not further distribute the material or use it for any profit-making activity or commercial gain

- You may freely distribute the URL identifying the publication in the public portal 


\title{
Permanent Magnet Thrust Bearings for Flywheel Energy Storage Systems - Analytical, Numerical, and Experimental Comparisons
}

\author{
Nikolaj A. Dagnaes-Hansen ${ }^{1}$ and IImar F. Santos ${ }^{1}$
}

\begin{abstract}
A new type of flywheel energy storage system uses a magnetic suspension where the axial load is provided solely by permanent magnets whereas active magnetic bearings are only used for radial stabilisation. This means that the permanent magnet bearing must provide all the axial damping. Furthermore, it must have as low a negative radial stiffness as possible to reduce the workload on the radial active magnetic bearings. Many different mathematical models for determining force, stiffness, and damping of permanent magnet bearings are available in the literature. This work will further develop the most applicable analytical and numerical methods in order to make them directly implementable for designing permanent magnet thrust bearings for flywheel energy storage systems. The outcome is a fast and efficient method for determining force, stiffness and damping when the bearing setup contains magnetic materials with relative permeability higher than one as well as when it does not. The developed method is validated against numerical and experimental results with good agreement.
\end{abstract}

\section{Keywords}

Flywheel energy storage, permanent magnet bearing

\section{Introduction}

Permanent magnet bearings (PMBs) provide poor damping and are unable to grant stable levitation on their own ${ }^{1}$. For commercial flywheel energy storage systems (FESS), they are thus often used in combination with active magnetic bearings (AMBs). This is the case in systems by e.g. Beacon Power $^{2}$ and Calnetix ${ }^{3}$ or the combined storage and attitude control systems (IPACS) developed by NASA ${ }^{45}$. Here, the flywheel is levitated by AMBs in five axes, while the PMBs are supplementing the AMBs by providing bias flux and additional lift force thus reducing the power consumption in the AMBs. However, the complexity and high cost of AMBs encourage reduction of the number of AMBs in the system and instead place a bigger role on the PMBs. This has led to a new type of FESS design where AMBs are only used in a radial direction whereas the axial lift is provided solely by PMBs. This simplified suspension is found in e.g. a FESS prototype at Uppsala University ${ }^{6}$, another prototype by Toh and $\mathrm{Chen}^{7}$, the patented design by the company WattsUp Power $^{89}$, and the patented design by the company Temporal Power ${ }^{10}$. This type of design does however pose certain challenges: First, in the absence of an axial active magnetic bearing, damping of axial vibrations is less straightforward because the permanent magnet bearing provides low to no damping. Second, if the permanent magnet thrust bearing is to carry the whole weight of the rotor and thus provide a positive stiffness in the axial direction, it will consequently provide a negative radial stiffness. This means that the radial $\mathrm{AMBs}$ must compensate the radial destabilising force coming from the PMB. Thus, it is of interest to reduce the negative radial stiffness coming from the PMB as much as possible. In summary, the main challenges of only using permanent magnets for axial levitation are to 1) ensure sufficient axial damping and 2) reduce radial negative stiffness coming from the PMB.

${ }^{1}$ Department of Solid Mechanics, Technical University of Denmark, Kgs. Lyngby, Denmark

Corresponding author:

IImar F. Santos, Technical University of Denmark, Tel.: +452562 69

Email: ifs@mek.dtu.dk 
One of the first to look into the design of modern repulsion type PMBs is Yonnet ${ }^{1112}$. Analytical maximization of stiffness to volume ratio has been dealt with by Marth et al. ${ }^{1314}$ for designs with negligible curvature - that is, they approximate circular magnets as long straight magnets making the problem planar instead of three-dimensional. A numerical investigation of the maximal radial stiffness, taking into account the curvature, is carried out by Moser et al. ${ }^{15}$ and by Lang and Lembke ${ }^{16}$ for the axisymmetric case and Bekinal and Jan ${ }^{17}$ in a general 3-dimensional case. An analytical minimization of the volume for a given load is dealt with by Beneden et al. ${ }^{18} 19$ for thrust bearings with and without back-iron; conic permanent magnet bearings have also been studied ${ }^{20}$.

In regards to damping, the modelling of electrodynamic bearings has intensively been studied at the Polytechnical University in Torino 212223 . Other important contributions to the study of eddy-current damping for magnetic bearings are made by Filatov and Maslen ${ }^{24}$ where a pure passive magnetic bearing is used to stabilise a flywheel and the work by Sandtner and Bleuler ${ }^{25} 26$ dealing with electrodynamic thrust bearings.

Experimental investigations of the performance and damping of PMBs have been carried out by NASA ${ }^{2728}$. They combine radial PMBs with an axial jewel bearing. An experimental investigation of a hybrid PMB-foil-bearing spinning up to $40 \mathrm{kRPM}$ is carried out by Bekinal et al. ${ }^{29}$. A 500 Wh FESS with radial PMBs and axial AMBs is experimentally investigated by Fremerey and $\mathrm{Kolk}^{30}$. A 1-DOF PMB with integrated electrodynamic dampers is experimentally investigated by Lembke ${ }^{31}$. The method developed by Hahn et al. ${ }^{32}$ provides a fast and efficient way to evaluate eddy-current damping for vibrating magnets. Their method is however only valid for cases where selfinductance can be neglected. They show that their method is in agreement with experimental results for magnets that can be approximated as dipoles. While axial damping is needed for safe operation of the flywheel, it is undesired to have any damping in the peripheral direction - that is, any drag on the spinning flywheel from the PMBs. Therefore, eddy-current losses in the peripheral direction have been investigated by Hedlund et al. ${ }^{33}$.

As mentioned above, a PMB with positive axial stiffness will unavoidably have a negative radial stiffness which will affect the radial stability. A way to mitigate this, without using AMBs, is by utilising gyroscopic effects as in the case of the Levitron ${ }^{3435}$. The stability of the Levitron is explained using a linear rotor-dynamic model by $\mathrm{Gasch}^{36}$ where the PMB axial and radial stiffness are numerically found. An experimental validation of the mathematical models describing the Levitron is carried out by Fujii ${ }^{37}$ and Simon et al. ${ }^{38}$.

Original Contribution As seen from the above section, the literature concerning PMB stiffness and damping is rich on well-established mathematical models as well as experimental validation methods. This work applies the established methods on the problem described above: how to design a permanent magnet thrust bearing ideal for FESS application, where it is crucial that axial damping is high and negative radial stiffness is close to zero. To ensure applicability of the methods, experimental validations of both force and damping calculations are carried out. Furthermore, in order to solve the problem at hand, some short-comings of the established methods were found. Therefore, the following are the novel contributions coming from this work:

- The common way to evaluate the stiffness of PMBs is to numerically find the change in the magnetic force for a small perturbation. This is a problematic approach for PMBs designed for FESS application. Here, PMB dimensions will typically be much larger than the perturbation. Therefore, the mesh in a 3-dimensional numerical solution will not be fine enough for an accurate stiffness estimation. Therefore, previous authors have sought other approaches, namely analytical and 2-dimensional solutions. In the numerical analysis carried out by Lang and Lembke ${ }^{16}$ they present a clever, computationally less resourceful method to evaluate both axial force and stiffness as well as radial stiffness by solving a 2-dimensional axisymmetric problem. However, they confine their investigation to cases where all materials in the system have relative permeability close to one. This is not likely the case in a real application where materials such as magnetic steel are present. Therefore, this work will investigate - analytically, numerically, as well as experimentally - the applicability of the method in cases where magnetic steel is present in the bearing configurations.

- The concept of stiffness can only be used if the forces are close to linear within the space that the flywheel rotor can move around in. Again, it is difficult to assess the linearity of the force using a 3-dimensional numerical method due to resolution problems in the mesh. Therefore, to accurately determine the radial forces even though the radial displacements are small compared to the bearing dimensions, an 
analytical method for obtaining the magnetic field will be presented. From this model, the bearing forces and stiffness are found. The results can be used to determine if the force is approximately linear and thus if it is actually a valid approximation to use the concept of stiffnesses. Furthermore, the analytical method will be experimentally validated.

- In regards to eddy-current, the method by Hahn et al. ${ }^{32}$ will be compared with experimental results for cases where the magnets cannot be approximated with dipoles. This has, to the best knowledge of the authors, not been done before. Furthermore, as mentioned before, the method is only valid if self-inductance is neglected. This work shows how to numerically assess if this assumption is valid in the current case.

Approach An analytical method for obtaining the magnetic field from which the bearing forces and stiffness are found is presented in Sec. . The analytical results are compared with experimental and numerical results. In Sec., the overall axial dynamics are assessed with the purpose of damping axial vibrations as much as possible. It is found that eddy-current damping is the most effective way to reduce vibrations. Therefore, a method to determine the eddycurrent forces is presented in Sec. . Results obtained using the method are compared to experimental and numerical results.

\section{Magnetic Fields and Forces}

\section{Theoretical Magnetic Field}

The magnets will be modelled by approximating the permanent magnets with thin sheets of current flowing on the surfaces of the magnets. For example, two solid cylinder magnets with magnetizations $\mathbf{M}$ pointing upwards will be modelled as seen in Fig. 1a. More complex configurations of circular magnets, such as the one in Fig. 1b, will consist of multiple cylindrical and annular surface currents. The magnetic field coming from the configuration will then be found by superpositioning the contribution from each current sheet. Analytical expressions for the magnetic field of current sheets have been available at least since the time of Maxwell ${ }^{39}$. For cylindrical and annular current sheets, solutions are derived by e.g. Snow ${ }^{40}$. For cylindrical current sheets, expressions are also made available by Kolbenheyer ${ }^{41}$ and further developed to the case of multiple sheets by Varga and Beyer ${ }^{42}$. For the general case of a conic cylinder, expressions are derived by L. K. Urankar ${ }^{43}$. His expressions simplify nicely in the special cases of a cylinder

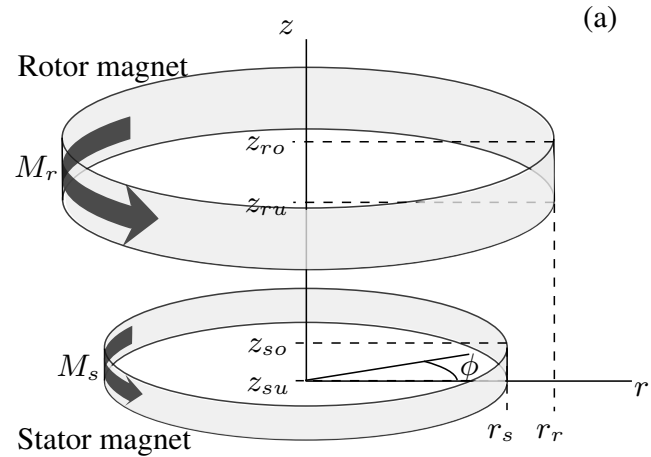

(b)

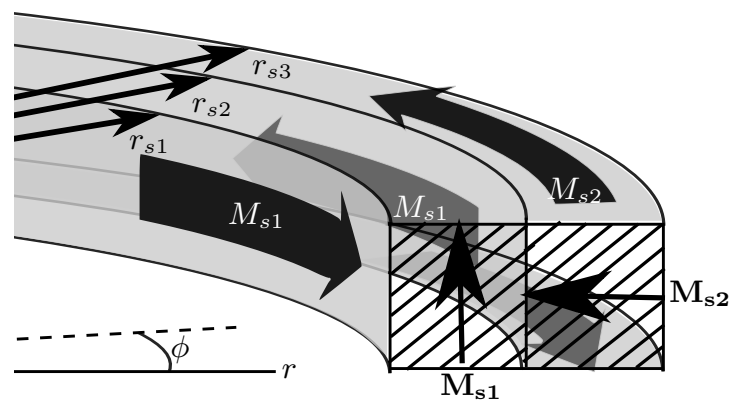

Figure 1. (a): Two disk-magnets modelled as thin cylinder sheets with magnetization $\mathbf{M}_{\mathbf{s}}=\left(0,0, M_{s}\right)^{T}$ and $\mathbf{M}_{\mathbf{r}}=\left(0,0, M_{r}\right)^{T}$. (b): Stator consisting of two ring magnets with magnetizations $\mathbf{M}_{\mathbf{s} \mathbf{1}}=\left(0,0, M_{s 1}\right)^{T}$ and $\mathbf{M}_{\mathbf{s 2}}=\left(-M_{s 2}, 0,0\right)^{T}$. Moment vectors are given in Cartesian coordinates and all moments result in currents $M_{r}, M_{s}$ flowing in a circumferential direction

and an annulus and will be used here. The magnetic potential $A_{\phi}$ and field density $\mathbf{B}=\left(B_{r}, 0, B_{z}\right)$ for a cylindrical sheet of radius $r^{\prime}$ and height $z_{1}^{\prime}-z_{0}^{\prime}$ are given by:

Cylinder:

$$
\begin{array}{r}
A_{\phi}=2 M_{s}\left(\frac { \gamma _ { 1 } } { 2 r } \left[\frac{\gamma_{1}^{2}+2 r^{\prime 2}+2 r^{2}}{a_{1}} K\left(k_{1}^{2}\right)-a_{1} E\left(k_{1}^{2}\right)\right.\right. \\
\left.-\frac{\left(r^{\prime}-r\right)^{2}}{a_{1}} \Pi\left(n_{3}^{2}, k_{1}^{2}\right)\right]-\frac{\gamma_{0}}{2 r}\left[\frac{\gamma_{0}^{2}+2 r^{\prime 2}+2 r^{2}}{a_{0}} K\left(k_{0}^{2}\right)\right. \\
\left.\left.-a_{0} E\left(k_{0}^{2}\right)-\frac{\left(r^{\prime}-r\right)^{2}}{a_{0}} \Pi\left(n_{3}^{2}, k_{0}^{2}\right)\right]\right) \times 10^{-7}
\end{array}
$$

$$
\begin{array}{r}
B_{r}(r, z)=2 M_{s}\left(\frac{a_{1}}{2 r}\left[\left(1+k_{1}^{\prime 2}\right) K\left(k_{1}^{2}\right)-2 E\left(k_{1}^{2}\right)\right]\right. \\
\left.-\frac{a_{0}}{2 r}\left[\left(1+k_{0}^{\prime 2}\right) K\left(k_{0}^{2}\right)-2 E\left(k_{0}^{2}\right)\right]\right) \times 10^{-7}
\end{array}
$$

$$
\begin{array}{r}
B_{z}(r, z)=2 M_{s}\left(\frac{\gamma_{1}}{a_{1}}\left[K\left(k_{1}^{2}\right)+\frac{r^{\prime}-r}{r^{\prime}+r} \Pi\left(n_{3}^{2}, k_{1}^{2}\right)\right]\right. \\
\left.-\frac{\gamma_{0}}{a_{0}}\left[K\left(k_{0}^{2}\right)+\frac{r^{\prime}-r}{r^{\prime}+r} \Pi\left(n_{3}^{2}, k_{0}^{2}\right)\right]\right) \times 10^{-7},
\end{array}
$$


where $E, K$, and $\Pi$ are the complete elliptic integrals of first, second, and third kind defined by eqs. (8) - (10), while:

$$
\begin{array}{rlrl}
\gamma & =z^{\prime}-z & a^{2}=\gamma^{2}+\left(r^{\prime}+r\right)^{2} & k^{2}=4 r r^{\prime} / a \\
k^{\prime 2}=1-k^{2} & n_{3}^{2}=4 r r^{\prime} /\left(r^{\prime}+r\right)^{2} .
\end{array}
$$

For an annular sheet with inner and outer radius $r_{0}^{\prime}$ and $r_{1}^{\prime}$ located at height $z^{\prime}$, we have:

Annulus:

$$
\begin{array}{r}
B_{r}(r, z)=2 \times 10^{-7} M_{s} \\
\times\left(\frac{\gamma}{a_{1} r}\left[2 r_{1}^{\prime} K\left(k_{1}^{2}\right)-\sum_{p=1}^{2}(-1)^{p} c_{1} \frac{r_{1}^{\prime} \pm c_{1}}{r \mp c_{1}} \Pi\left(n_{p, 1}^{2}, k_{1}^{2}\right)\right]\right. \\
\left.-\frac{\gamma}{a_{0} r}\left[2 r_{0}^{\prime} K\left(k_{0}^{2}\right)-\sum_{p=1}^{2}(-1)^{p} c_{0} \frac{r_{0}^{\prime} \pm c_{0}}{r \mp c_{0}} \Pi\left(n_{p, 0}^{2}, k_{0}^{2}\right)\right]\right),
\end{array}
$$

$$
\begin{aligned}
B_{z}(r, z)=2 \times 10^{-7} M_{s} & \left(\left[2 g_{1}-2 \frac{r_{1}^{\prime}}{a_{1}} K\left(k_{1}^{2}\right)\right]\right. \\
- & {\left.\left[2 g_{0}-2 \frac{r_{0}^{\prime}}{a_{0}} K\left(k_{0}^{2}\right)\right]\right), }
\end{aligned}
$$

where the upper sign corresponds to $p=1$ and the lower to $p=2$ and

$$
\begin{array}{r}
c^{2}=\gamma^{2}+r^{2} \quad n_{p}^{2}=\frac{2 r}{r \mp c} \\
g=\int_{0}^{\frac{\pi}{2}} \sinh ^{-1}\left(\frac{-|\gamma|}{\sqrt{r^{\prime 2}+r^{2}-2 r r^{\prime} \cos \alpha}}\right) \mathrm{d} \alpha,
\end{array}
$$

where the integral $g$ is numerically solved. The elliptic integrals are defined as

$$
\begin{array}{r}
K\left(k^{2}\right)=\int_{0}^{1}\left[\left(1-t^{2}\right)\left(1-k^{2} t^{2}\right)\right]^{-\frac{1}{2}} \mathrm{~d} t \\
E\left(k^{2}\right)=\int_{0}^{1}\left(1-t^{2}\right)^{-\frac{1}{2}}\left(1-m^{2} t^{2}\right)^{\frac{1}{2}} \mathrm{~d} t \\
\Pi\left(n^{2}, k^{2}\right)=\int_{0}^{\frac{\pi}{2}} \frac{\mathrm{d} \theta}{\left(1-n^{2} \sin ^{2} \theta\right) \sqrt{1-k^{2} \sin ^{2} \theta}}
\end{array}
$$

In fig. 2, the eqs. (2), (3), (5), and (6) are used to calculate the field of some basic configurations. Fig. 2a shows the magnetic field density of a cylinder sheet. Fig. 2b shows the magnetic field density of an annular sheet. In Fig. 2c, multiple annular and cylinder sheets are combined to form a Halbach array. Here it is seen how the magnetic field is augmented above the magnet and cancelled below as expected.

\section{Theoretical Magnetic Force}

Having found the magnetic field from the stator part of the $\mathrm{PMB}$, the forces on the rotor $\mathbf{F}=\left(F_{x}, F_{y}, F_{z}\right)$ can now be obtained for a rotor placed in any position. This is done using the Lorentz Force Law which is integrated numerically over the rotor sheet area $A_{s}$ :

$$
\mathbf{F}=\int \mathbf{M} \times \mathbf{B} \mathrm{d} A_{s}
$$

When the stator and rotor are coaxially aligned, the rotor is at equilibrium and some elegant analytical solutions are made available by Lang $^{44}$ for a cylindrical sheet:

$$
\begin{aligned}
F_{z} & =2 \pi r_{r} M_{r}\left[A_{\phi}\left(r_{r}, z_{r o}\right)-A_{\phi}\left(r_{r}, z_{r u}\right)\right], \\
k_{z} & =2 \pi r_{r} M_{r}\left[B_{r}\left(r_{r}, z_{r o}\right)-B_{r}\left(r_{r}, z_{r u}\right)\right], \\
k_{r} & =-\frac{1}{2} k_{z}
\end{aligned}
$$

where $k_{z}$ is the axial stiffness and $k_{r}=k_{x}=k_{y}$ is the radial stiffness and $M_{r}$ is current per surface area in a circumferential direction. Eq. (14) is not only valid for cylindrical sheets but for all magnets placed in a static field where no free currents are present. In the literature, it is commonly stated that Earnshaw's theorem leads to $k_{r} \leq-\frac{1}{2} k_{z}$. To avoid discussion, a derivation of the more specific $k_{r}=-\frac{1}{2} k_{z}$ is given in the appendix. The relation is important because it shows that we cannot increase $k_{z}$ without also increasing $-k_{r}$. Also, it means that we can find both $k_{z}$ and $k_{r}$ by solving an axisymmetric problem which is significantly less demanding than the straight-forward way of determining $k_{r}$ : by looking at the radial force for a 3 dimensional problem with a radially displaced rotor.

\section{Experimental Magnetic Force}

One of the assumptions of the above expressions is that all materials have a relative permeability of 1 . This can be difficult to obey in practice, and therefore it is of interest to assess the size of the error between theory and practice in the presence of materials with high permeability. The test setup in Fig. 3 is used to obtain experimental results for comparison with the theoretical axial and radial magnetic forces. Three different bearing configurations, all including steel with high permeability, have been tested. Each of their respective cross sections can be seen in Fig. 4. Bearing configuration type 1 consist of arrays of $3 \mathrm{~mm}$ cube magnets whereas bearing type 2 and type 3 both consist of ring magnets. The rotor is rotating with 95 RPM and the force is obtained as a mean of measurements for one whole rotation. 


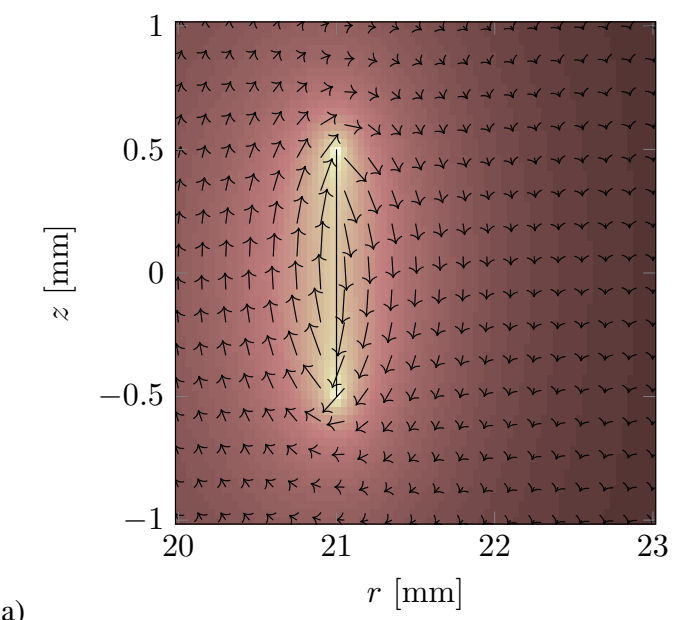

(a)

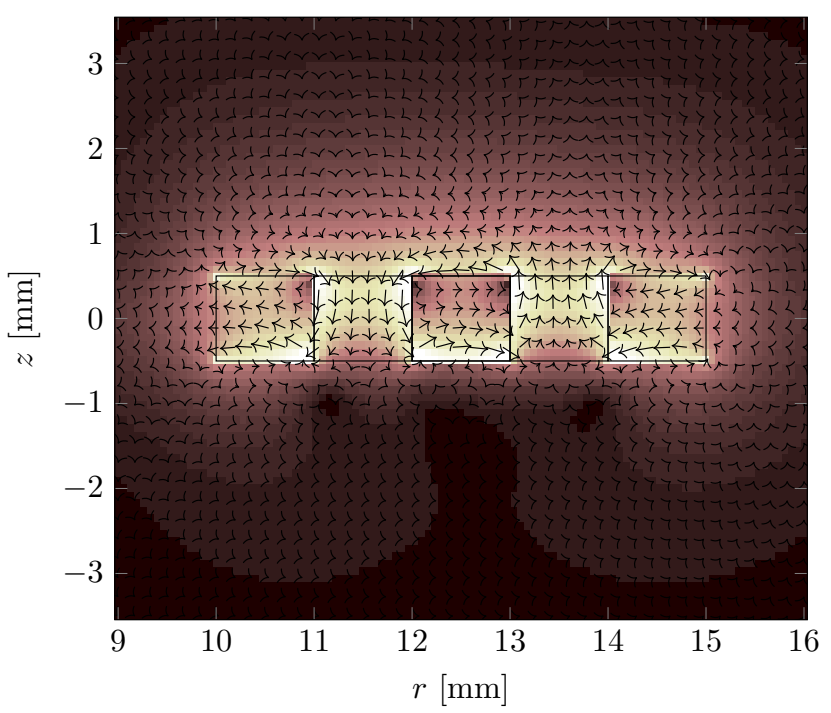

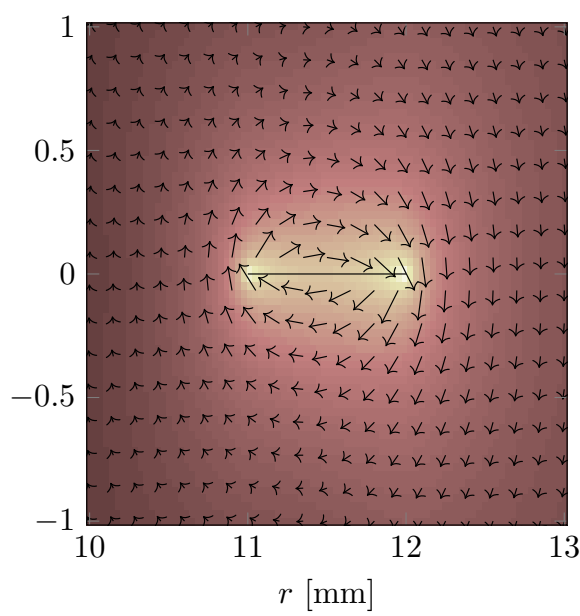

(b)

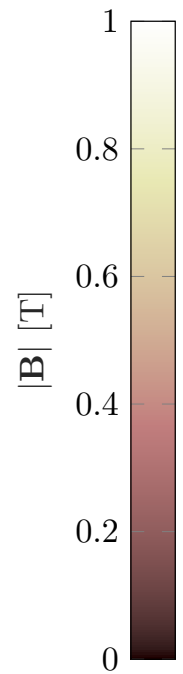

(c)

Figure 2. Magnetic field density B from: (a) cylindrical sheet, (b) annular sheet, and (c) a Halbach array composed of sheets. All currents have magnitude $M_{s}=955 \mathrm{kA} / \mathrm{m}$.

The measurements have been carried out at different $z$ and $y$-positions of the rotor.

The results for the three different configurations can be seen in Fig. 5, 6, and 7. For the theoretical results, the axial force $F_{z}$ has been found analytically using Eq. 12 whereas the radial force $F_{r}$ has been found numerically using Eq. 11 based on an analytically obtained B from eq. (2) and (3). Thus the method for finding the radial force will be referred to as semi-analytical. Numerical values for all parameters can be found in the appendix. For $F_{z}$, the results obtained using the program FEMM ${ }^{45}$ are included. It can be seen that the analytical results and the FEMM results are identical when the steel is not included in the FEMM calculations. It can also be seen that there is a significant difference in $F_{z}$ when the steel is accounted for. The bearings are designed for a load between 20 and $40 \mathrm{~N}$. In this interval, the maximum relative error $e=\frac{F_{z, F E M M}-F_{z, E x p}}{F_{z, E x p}}$ between theoretical and experimental results, when the steel is not accounted for, is $22 \%, 28 \%$, and $22 \%$ for bearing type 1, 2, and 3 respectively. When the steel is accounted for, the error is reduced to $6 \%, 7 \%$, and $8 \%$ respectively.

A rough comparison of the computation times for evaluating $F_{z}$ is shown in Tab. 1. The computations are carried out in MatLab interfacing with FEMM using OctaveFEMM $^{45}$. The calculations are carried out on a standard laptop with an i7 $2.60 \mathrm{GHz}$ processor. The table shows that the analytical method is almost ten times faster than FEMM when no steel is accounted for. For bearing type 1 however, the computation time is only around three times faster. This configuration consists of more current sheets and thus a higher workload for the analytical method. Hence, as the configuration becomes more complex, the difference in computation times is decreasing. If steel is accounted for in FEMM, the computation times are approximately doubled. However for all cases, the computation times are low which proves the applicability of both methods. If one is to carry out many computations, for example in the case of an optimization procedure, the lower computation time of the analytical method may be of significant advantage. 


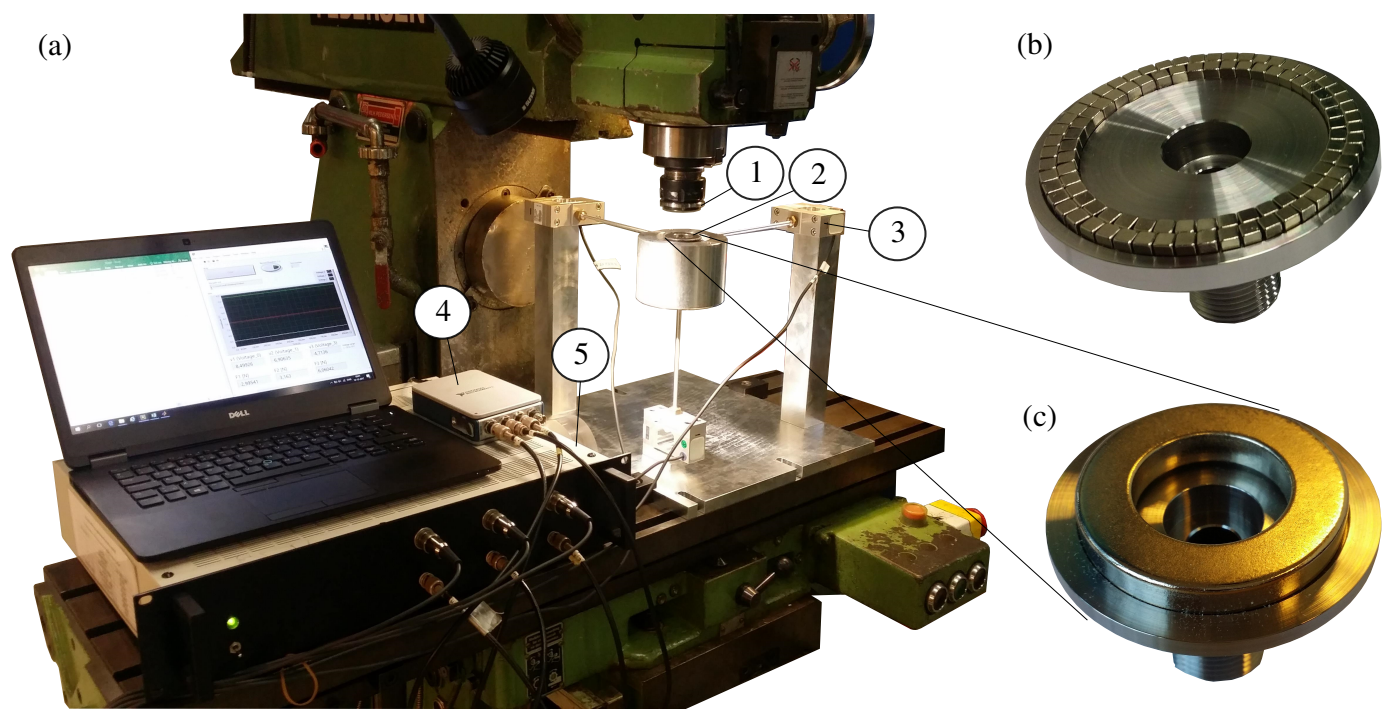

Figure 3. (a): Measurement of PMB forces. (1): Rotor Part of PMB. (2): Stator Part of PMB (3): Force transducer, HBM S2M. (4): Data acquisition, NI 9215. (5): Amplifier, HBM AE301. (b): Stator part of bearing type 1. (c): Stator part of bearing type 2.

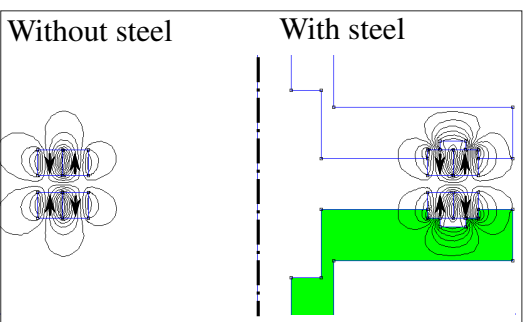

(a) Type 1

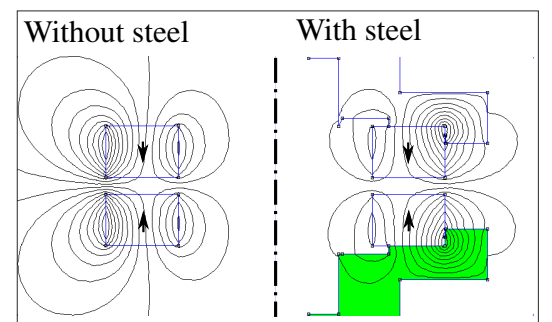

(b) Type 2

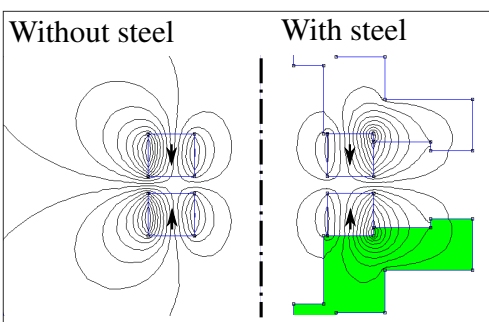

(c) Type 3

Figure 4. Cross-section of PMB in experimental set-up. The direction of the magnetization is shown as arrows. The contours show the magnetic field. The steel part of the stator is filled with color. It has relative permeability $\mu_{r}=410$. It is seen how the steel is concentrating the flux around the magnets while the flux is more spread out when no steel is present.

\begin{tabular}{c|ccc} 
& Mean & St.dev. & Max. rel. error \\
Type 1, analytical calc. & $0.17 \mathrm{~s}$ & $0.02 \mathrm{~s}$ & $22 \%$ \\
Type 2, analytical calc. & $0.07 \mathrm{~s}$ & $0.01 \mathrm{~s}$ & $28 \%$ \\
Type 3, analytical calc. & $0.06 \mathrm{~s}$ & $0.008 \mathrm{~s}$ & $22 \%$ \\
\hline Type 1, FEMM no steel & $0.58 \mathrm{~s}$ & $0.02 \mathrm{~s}$ & $22 \%$ \\
Type 2, FEMM no steel & $0.66 \mathrm{~s}$ & $0.04 \mathrm{~s}$ & $28 \%$ \\
Type 3, FEMM no steel & $0.55 \mathrm{~s}$ & $0.03 \mathrm{~s}$ & $22 \%$ \\
\hline Type 1, FEMM w/ steel & $1.34 \mathrm{~s}$ & $0.05 \mathrm{~s}$ & $6 \%$ \\
Type 1, FEMM w/ steel & $1.47 \mathrm{~s}$ & $0.08 \mathrm{~s}$ & $7 \%$ \\
Type 1, FEMM w/ steel & $1.19 \mathrm{~s}$ & $0.04 \mathrm{~s}$ & $8 \%$
\end{tabular}

Table 1. Comparison of the time it takes to evaluate $F_{z}$ in one point. The results show the mean and standard deviation for the computation times for the results plotted in Fig. 5a, 6a, and 7a respectively. The maximum relative error wrt. experimental results is given in the table as well.

It is important to note that the presence of steel only results in a bearing with a larger load capacity. This means that one should not fear over-estimating the load carrying capacity of the bearing by not accounting for the steel. One way to compensate for the under-estimation made by the analytical expressions, is to make the magnetization slightly larger than in reality. According to the data sheet of the magnets used for the experiments, the magnetization can vary between $860-955 \mathrm{kA} / \mathrm{m}$. It was found that if the magnetization was increased to $955 \mathrm{kA} / \mathrm{m}$, which is the upper limit of the actual magnetization, the analytical equations are in good agreement with the experimental results. This can be seen for the radial force in Fig. 5b, 6b, and $7 b$. Here it is demonstrated how the semi-analytical method is useful for determining radial forces. This shows how the radial magnetic force in a 3-dimensional case can be calculated in a fast and efficient way - especially if no steel is present because the method can be applied without any adjustment. Even when steel is present and one needs to artificially adjust the magnetization from $883.31 \mathrm{kA} / \mathrm{m}$ to $955 \mathrm{kA} / \mathrm{m}$, the semi-analytical method is still a useful tool because it has a low computational time and can yield accurate results even for small relative displacements. This is more cumbersome to achieve with a numerical solver in a 3-dimensional case because of the large dimensions of the bearing compared to the smaller dimensions of the rotor displacements which demands agreater effort in proper meshing. This is demonstrated using a tutorial example from the commercial software COMSOL ${ }^{46}$. The example is made by COMSOL specifically for demonstrating how to calculate radial stiffness and force for a radial PMB, thus it is assumed that the example is set up optimally for performing this 


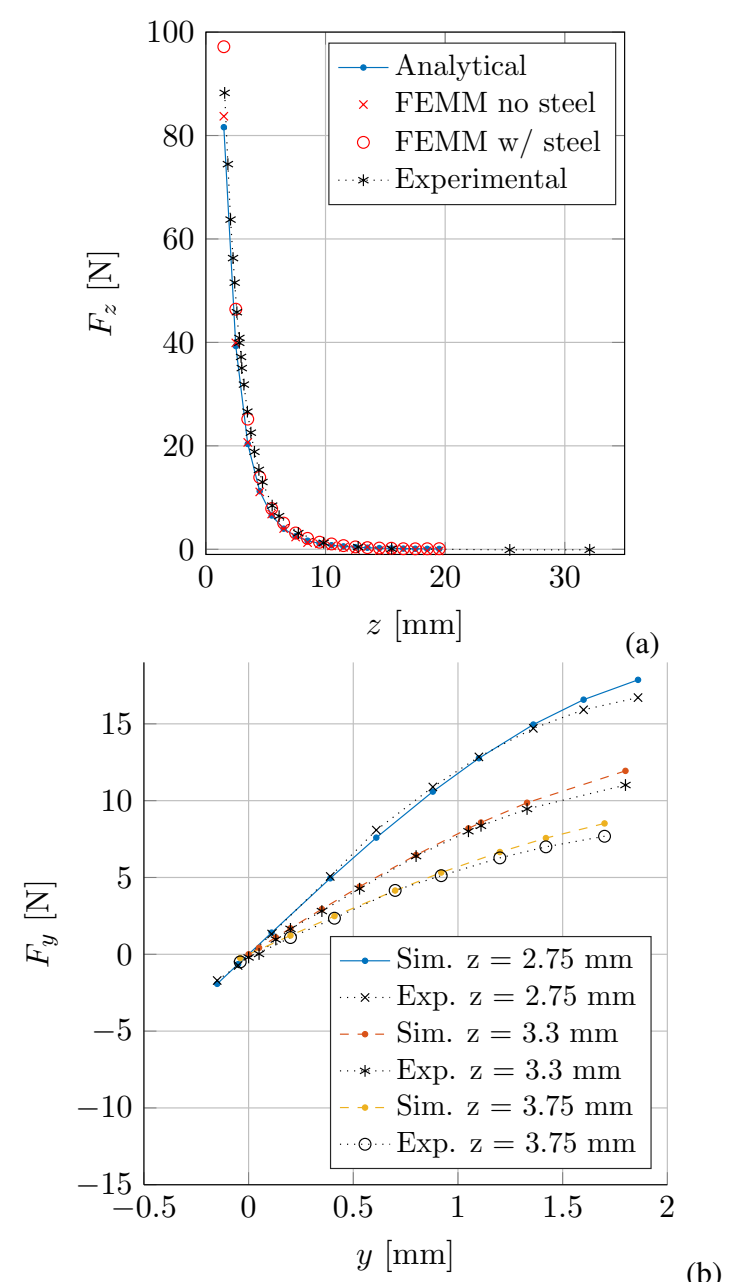

Figure 5. Comparison of experimentally and numerically obtained forces in bearing type 1. (a): Axial force. magnetization for analytical calculations is $883.31 \mathrm{kA} / \mathrm{m}$. (b): Radial force. magnetization for semi-analytical calculations is adjusted to $955 \mathrm{kA} / \mathrm{m}$.

task. A cross-section of the bearing used in the example can be seen in Fig. 8 along with the simulated magnetic flux density. The radial force is calculated in COMSOL and compared with the radial force calculated using the semianalytical method in Fig. 9. For large rotor displacements, Fig. 9a, the COMSOL results are seen to have approximately converged when using the normal mesh. The converged results are in agreement with the semi-analytical results. The computation time for the force evaluation in a single point is on average $7 \mathrm{~s}$ for the semi-analytical method and 48 $\mathrm{s}$ when using COMSOL and the normal sized mesh. For small rotor displacements, Fig. 9b, the COMSOL results are seen to have difficulties converging unless an extra fine volumetric and surface mesh is used. Only for the extra fine mesh are the results in agreement with the semi-analytical results. The example serves to indicate two benefits from the semi-analytical method: 1) it is straight-forward to obtain accurate results for small radial displacements, and 2) the computation time is low.
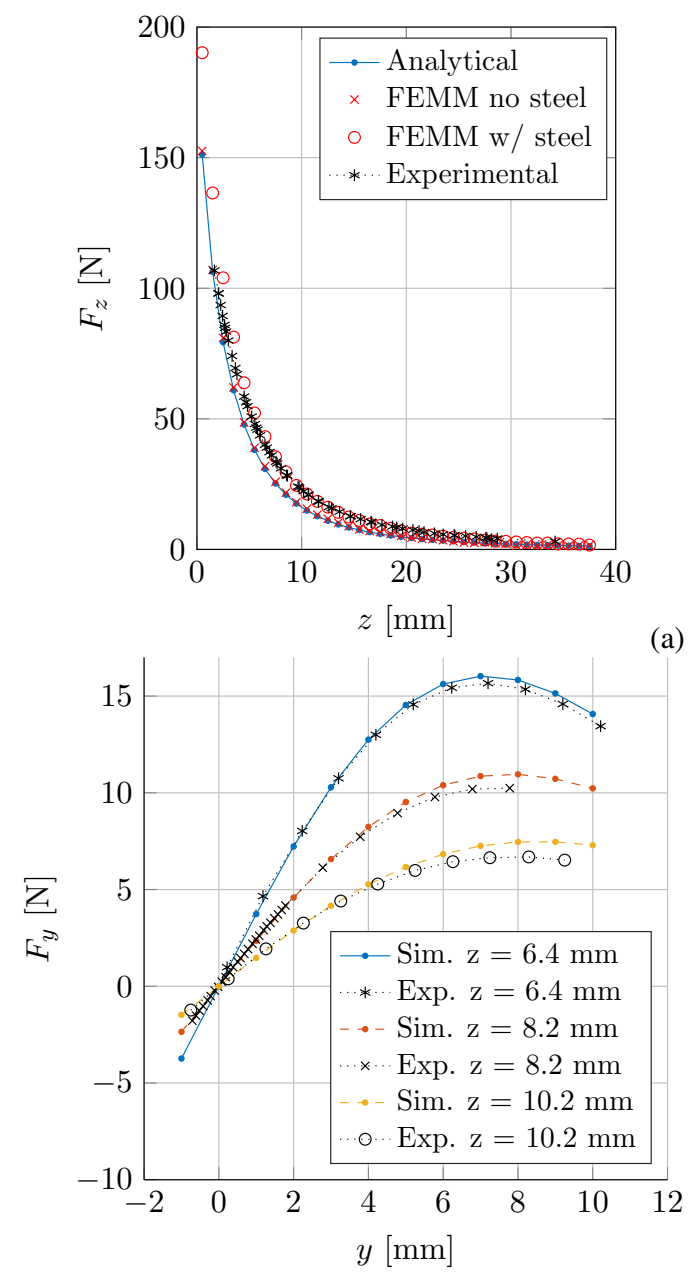

(a)

(b)

Figure 6. Comparison of experimentally and numerically obtained forces in bearing type 2. (a): Axial force. magnetization for analytical calculations is $883.31 \mathrm{kA} / \mathrm{m}$. (b): Radial force. magnetization for semi-analytical calculations is adjusted to $955 \mathrm{kA} / \mathrm{m}$.

The results from Fig. 5, 6, and 7 also provide insight into the linearity of the forces. If the forces are non-linear even for small displacements, the stiffness coefficients become inaccurate. In the radial direction, the free movement of the flywheel is limited by backup-bearings with a clearance typically around $100-300 \mu \mathrm{m}$. From the figures, it is seen that the radial force is approximately linear at least up to 0.5 $\mathrm{mm}$. Therefore, it can be concluded that the radial stiffness coefficient is applicable.

\section{Axial rotor vibrations}

To damp axial vibrations, two possible dampers are suggested as seen in Fig. 10: a visco-elastic damper (12) and an eddy-current damper (ECD) (9) (10. The visco-elastic damper cannot work directly between rotor and stator and is thus placed between stator and housing. A mechanical model of the axial dynamics can be seen in Fig. 10b. The objective of the axial suspension is to make sure that the rotor and 


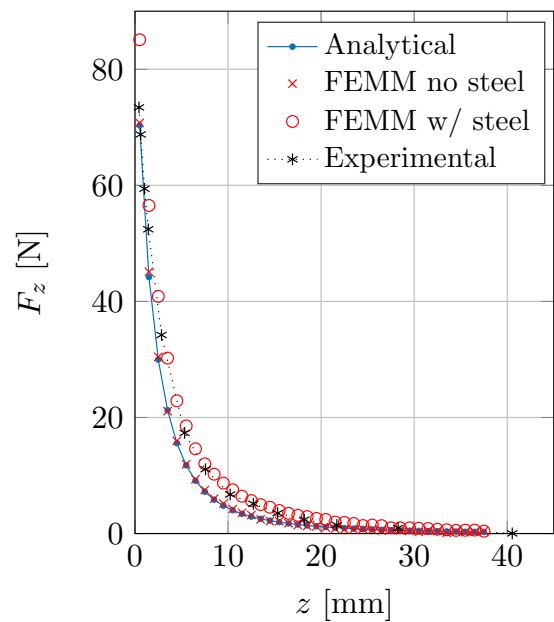

(a)

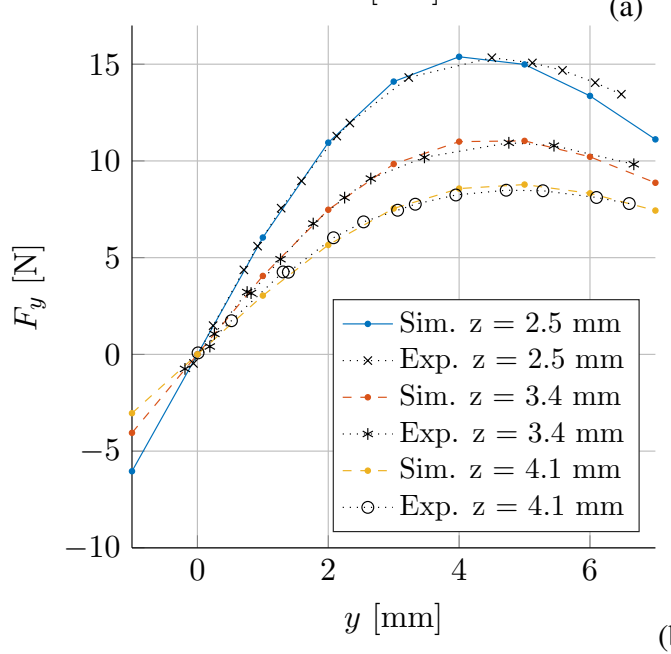

(b)

Figure 7. Comparison of experimentally and numerically obtained forces in bearing type 3. (a): Axial force. magnetization for analytical calculations is $883.31 \mathrm{kA} / \mathrm{m}$. (b): Radial force. magnetization for semi-analytical calculations is adjusted to $955 \mathrm{kA} / \mathrm{m}$.

$\mathrm{XO}(2)=0.001 \mathrm{~mm}$ Volume: Magnetic flux density norm (T) Arrow Surface: Magnetic flux density (Material)

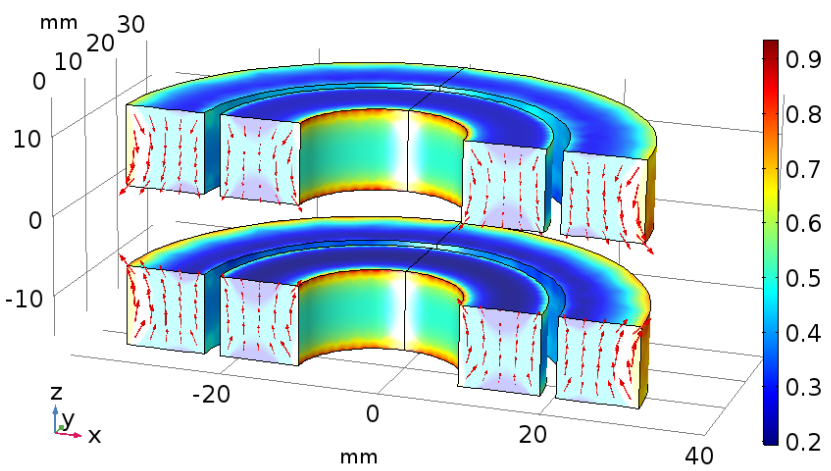

Figure 8. A slightly modified COMSOL example of a radial PMB downloaded from the internet ${ }^{46}$. The modifications consist of removing the rotor middle magnet which had a magnetization pointing radially outwards and the stator middle magnet which had a magnetization pointing radially inwards. This has been done to better obtain numerical convergence. Furthermore, all fillets applied on the magnet edges have been removed for easier comparison with the analytical method. Finally, the magnetization of the magnets has been modified to consist of a magnetization of $955 \mathrm{kA} / \mathrm{m}$.
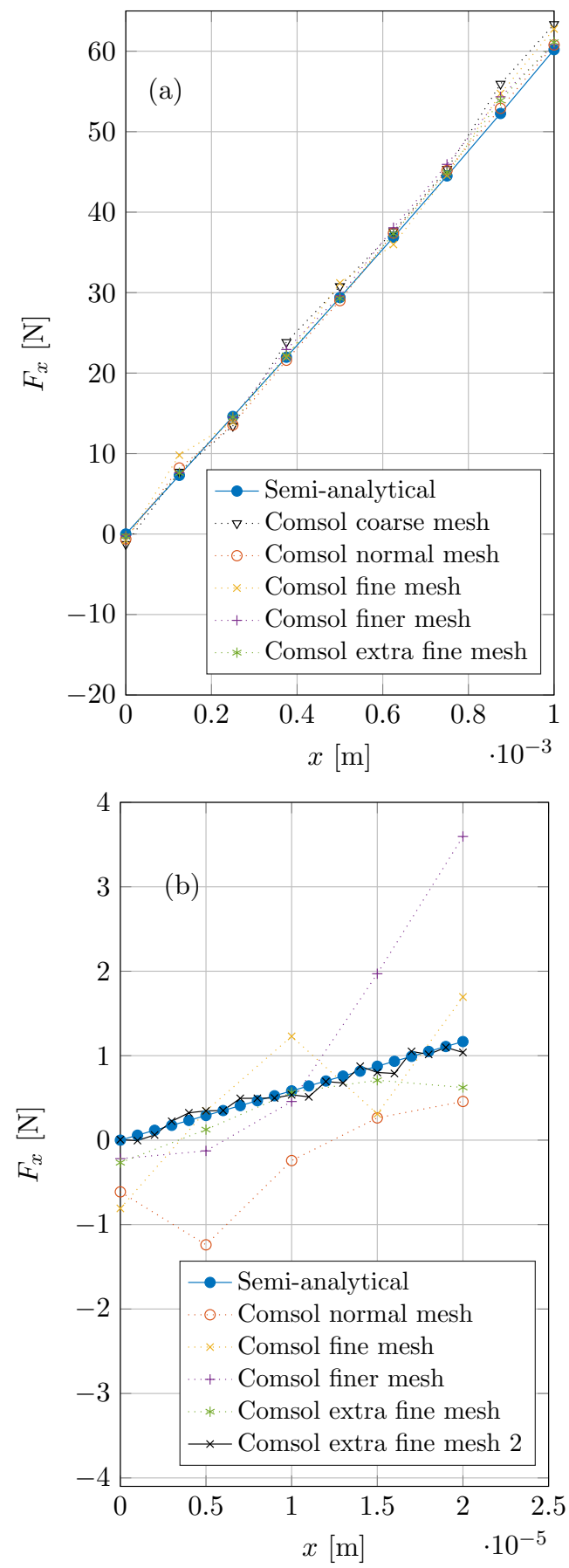

Figure 9. Radial force of the example shown in Fig. 8. Other than the modifications described in Fig. 8, the general tetrahedral mesh size has been changed according to the figure legend. For the semi-analytical method, the Lorentz force law has been evaluated numerically using $n_{z}=8$ points axially for each current sheet and $n_{\phi}=10$ points circumferentially. (a): Large radial displacement. (b): Small radial displacement. The graph called 'Comsol extra fine mesh 2' has, in addition to the extra fine mesh, a triangular mesh on the rotor surface which is refined from a maximum size of $1.5 \mathrm{~mm}$ to a maximum size of $0.5 \mathrm{~mm}$.

stator do not touch when subject to outer perturbations. We assume that the outer perturbations consist of the housing vibrating sinusoidally with amplitude $z_{h}$ and frequency $\Omega_{h}$. If we first fix the stator to the housing such that only the rotor is moving, we obtain, using Newton's equation, the following 


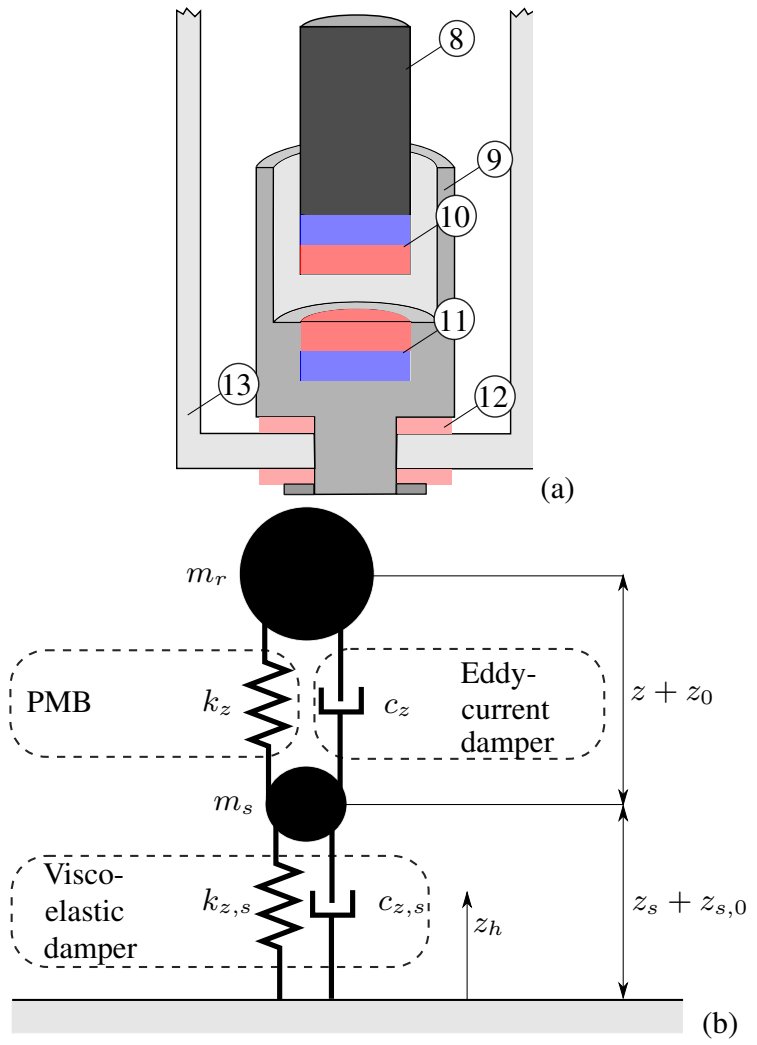

Figure 10. (a): Cross-section of PMB in experimental set-up. (8): Rotor. (9): Tube with high electrical conductivity. (10): Rotor magnet for combined PMB and ECD. (11): Stator part of PMB. (12): Visco-elastic damper. (13): Housing. (b): Mechanical 2-degrees-of-freedom model for axial vibrations. The stator part of the PMB is modelled as a particle with mass $m_{s}$ and the rotor has mass $m_{r}$.

relation

$$
\frac{z}{z_{h}}=\sqrt{\frac{1+(2 \zeta r)^{2}}{\left(1-r^{2}\right)^{2}+(2 \zeta r)^{2}}}
$$

(no movement of stator: 1-DOF system),

where $r=\Omega_{h} / \sqrt{k_{z} / m_{r}}, \quad \zeta=c_{z} /\left(2 m_{r} \omega_{n}\right)$, and $\omega_{n}=$ $\sqrt{k_{z} / m_{r}}$.

The maximum value of Eq. (15) for $\Omega_{h}$ varied around the resonance frequency is plotted as a function of $k_{z}$ and $c_{z}$ in Fig. 11a. It can be seen that an increase in PMB stiffness $k_{z}$ increases the vibration amplitude. This is interesting in view of the relation between axial and radial PMB stiffness, $k_{r}=-\frac{1}{2} k_{z}$. It shows that if $k_{z}$ is low, we obtain both a $k_{r}$ closer to zero and small vibrations of $z$ which are both desirable features. The stiffness does however not influence the amplitude much compared to the eddy-current damping $c_{z}$ which can be seen to most effectively decrease the vibration amplitude. Thus, we need as high an eddy-current damping $c_{z}$ as possible to reduce the vibrations of $z$.
If we include the movements of the stator, we get:

$$
\frac{z}{z_{h}}=\left|\frac{\left(k_{z, s}+i \Omega_{h} c_{z, s}\right) m_{r} \Omega_{h}^{2}}{P_{4} \Omega_{h}^{4}+P_{3} i \Omega_{h}^{3}+P_{2} \Omega_{h}^{2}+P_{1} i \Omega_{h}+k_{r} k_{s}}\right|
$$

(incl. movement of stator: 2-DOF system),

where $P_{4}=m_{r} m_{s}, P_{3}=-\left(\left(c_{z}+c_{z, s}\right) m_{r}+c_{z} m_{s}\right), P_{2}=$ $-\left(\left(k_{r}+k_{s}\right) m_{r}+c_{r} c_{s}+k_{r} m_{s}\right)$, and $P_{1}=\left(c_{r} k_{s}+c_{s} k_{r}\right)$. The maximum value of Eq. (16) for $\Omega_{h}$ varied around the two resonance frequencies is plotted as a function of $k_{z, s}$ and $c_{z, s}$ in Fig. $11 \mathrm{~b}$. It can be seen clearly that increased visco-elastic damping $c_{z, s}$ and stiffness $k_{z, s}$ will result in an increased vibration. Thus, the suspension between stator and housing should be designed with a low stiffness $k_{z, s}$ and as little damping $c_{z, s}$ as possible.

In summary, the most effective way to isolate the vibrations of $z$ is by making a suspension with low $k_{z, s} c_{z, s}$, and the only way to dampen the vibrations is by increasing $c_{z}$. This raises the question of how large the eddy-current damping is actually able to get without making the damper too large for practical application. This question will be assessed in the following section.

\section{Eddy-current Damping}

In Fig. 10a, the stator tube surrounding the rotor magnet functions to dissipate energy as eddy-currents. The magnitude of the damping force $F_{E C D}$ can be expressed as a function of the field density found in Sec. . It is assumed that all materials in the system have relative permeability of 1 , that the eddy-currents are only occurring in the stator tube (9), and that self-inductance caused by the induced eddycurrents can be neglected. The current density is defined as $\mathbf{J}$, conductivity as $\sigma$, the electric field as $\mathbf{E}$, and the volume of the stator tube as $V$. Combining Ohm's law

$$
\mathbf{J}=\sigma \mathbf{E},
$$

Faraday's law

$$
\mathbf{E}=\dot{z} \times \mathbf{B}
$$

and the Lorentz force law

$$
\mathbf{F}_{\mathbf{E C D}}=\int \mathbf{J} \times \mathbf{B} \mathrm{d} V
$$

the following expression for the $z$-component of the damping force is obtained ${ }^{32}$ :

$$
F_{E C D}=\int_{r_{0}}^{r_{1}} \int_{z_{l}}^{z_{u}} 2 \pi r \sigma \dot{z} B_{r}^{2}(r, z) \mathrm{d} z \mathrm{~d} r .
$$



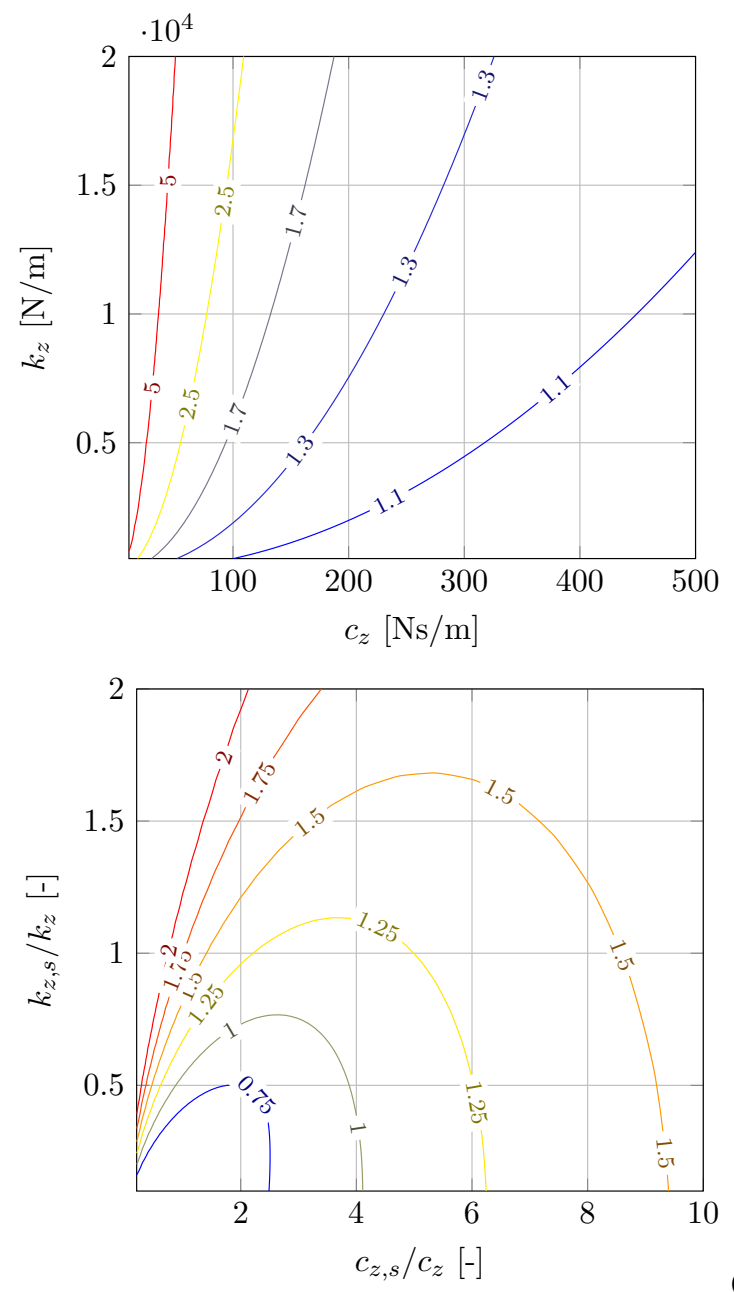

(a)

(b)

Figure 11. (a): Maximum value of $z / z_{h}$ for the 1-DOF system in Eq. (15). (b): Maximum value of $z / z_{h}$ for the 2-DOF system in Eq. (16). $k_{z}=5 \mathrm{kN} / \mathrm{m}, c_{z}=50 \mathrm{~N} /(\mathrm{m} / \mathrm{s})$.

Where $r_{0}$ and $r_{1}$ are the inner and outer diameter of the tube, and $z_{l}$ and $z_{u}$ are the coordinates of the tube ends. The damping coefficient is then readily found for small vibrations as

$$
c_{z}=\frac{F_{E C D}}{\dot{z}}=\int_{r_{0}}^{r_{1}} \int_{z_{l}}^{z_{u}} 2 \pi r \sigma B_{r}^{2}(r, z) \mathrm{d} z \mathrm{~d} r .
$$

This expression can be used for most realistic axial damper configurations for flywheels - also if the magnets are placed as rings on the outside of the tube which would be the obvious choice for most flywheel batteries which commonly consist of hollow flywheel hubs. The double integration is done numerically. For the results presented in Fig. 14, the authors experienced a converged solution when using 30-60 integration points in the $z$-direction and 2-5 integration points in the $r$-direction corresponding to a calculation time of around $1 \mathrm{~s}$. If the thickness of the tube is small, the expression can be simplified further to only involve integration in the $z$-direction. This has been done by Hahn et al. ${ }^{32}$ with great success. They compare their theoretical expression with experimental results with good agreement. Their agreement between experiments and theory show the usefulness of eq. (21). However, they only look at disk magnets that can be approximated with dipoles. Also, as described by Detoni et al. ${ }^{47}$, the self-inductance can dramatically decrease the effect of the induced eddy-currents - from enacting viscous damping at lower frequencies to losing the damping effect at high frequencies and instead work as a mechanical spring and thus contribute to the axial stiffness. To ensure that the self-inductance is in fact negligible, one can numerically solve the Maxwell's Equations. To ensure the validity of eq. (21) for other cases than dipoles and to ensure that self-inductance is negligible, an experimental investigation and a numerical finite element (FE) analysis have been carried out. The test setup is sketched in Fig. 12. A rotor is levitated between two active magnetic bearings (AMBs) which are holding the rotor in place radially whereas a permanent magnet bearing is placed in the bottom to carry the axial load. An eddy-current damper (ECD) consisting of a magnet on the rotor and a tube on the stator/housing is present in the top. The magnet used for eddy-current damping has radius $r_{e}$ and height $h_{e}$. The tube has inner radius $r_{0}$ and outer radius $r_{1}$. The tube is not covering the magnet completely as seen in Fig. 12c. The uncovered part of the magnet is denoted with length $z_{\text {cover }}$ as seen in Fig. 12a. First, it is investigated how the tube self-inductance is affecting the damping. This is done using the results from an FE analysis conducted in COMSOL seen in Fig. 13. The damping ratio $\zeta$ is plotted for varying $\dot{z}$. As seen, the damping ratio decreases for high speeds as expected. The damping ratio is decreased by $3 \%$ at $5.5 \mathrm{~m} / \mathrm{s}$ for the long magnet and $7.8 \mathrm{~m} / \mathrm{s}$ for the short one. For the test setup, the natural frequency in the axial direction is 15.5 $\mathrm{Hz}$ and the maximum vibration is $1 \mathrm{~mm}$ which corresponds to a maximum velocity of $0.017 \mathrm{~m} / \mathrm{s}$, much lower than the speeds where the $3 \%$ reduction occurs. It can thus be concluded that the self-inductance is negligible. Therefore, we will proceed to compare the damping ratio obtained from eq. (21) with the damping ratio obtained using COMSOL (at low speeds) and using experiments. This is done in Fig. 14. As seen, the COMSOL results are in good agreement with eq. (21). Also, the theoretical results are within the $95 \%$ confidence interval of the experimental results for all but one point. There is a clear general trend of the theoretical results yielding a higher damping ratio than the experimental results. The maximum relative error between theoretical and experimental results, $e=\frac{\left|\zeta_{\text {theo }}-\operatorname{mean}\left(\zeta_{\text {exp }}\right)\right|}{\operatorname{mean}\left(\zeta_{\text {exp }}\right)}$ is on average 9 $\%$ with a maximum of $15 \%$ for the case $h_{e}=2 \mathrm{~cm}$ and $z_{\text {cover }}=10 \mathrm{~mm}$. The deviation is due to the magnetization 


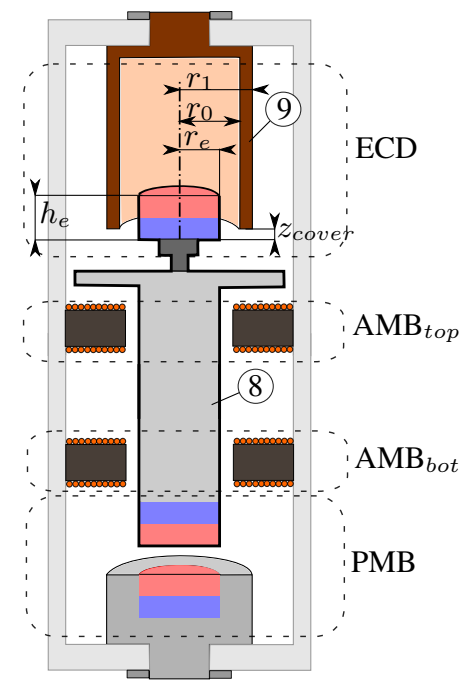

(a)

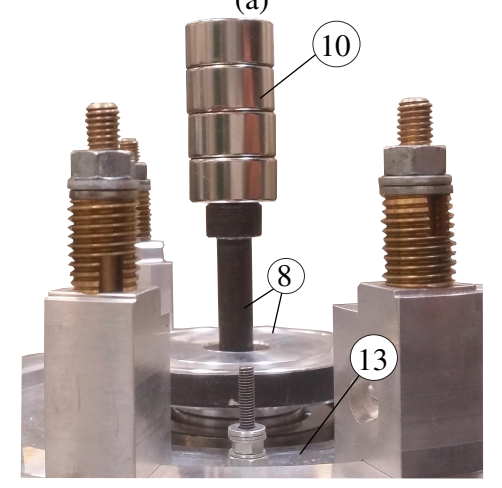

(b)

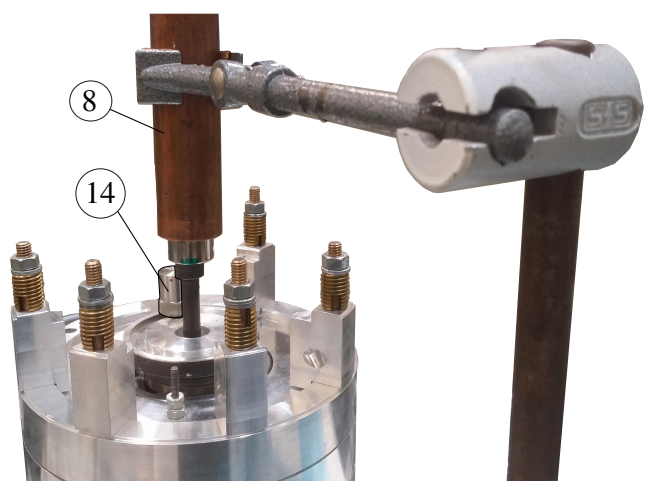

(c)

Figure 12. (8): Rotor. (9): Copper tube. (10): Rotor magnets for ECD. (13): Housing. (14): Accelerometer, Brüel \& Kjær 1366. (a): Sketch. (b): Setup without tube. (c): Setup with tube.

$M_{s}$ and the electric conductivity, $\sigma$ which are subject to uncertainties. The deviation is however acceptable and it can be concluded that the method is useful for determining the damping ratio in our case.

\section{Conclusion}

This article provides a practical method for designing permanent magnet thrust bearings for flywheel energy storage systems.
It was demonstrated how the load carrying capacity as well as radial and axial stiffness can be found in a fast and efficient way using analytical expressions. If the bearing configuration includes materials with high permeability, then the analytical method is underestimating the load carrying capacity by $22 \%-28 \%$ from conducted experiments. In order to reduce the error, two approaches are presented. One can either choose to include the magnetic materials in a numerical solver such as FEMM which also provides a fast and efficient way to find the force in the axisymmetric case. This reduced the error to $6 \%-8 \%$. Another option is to increase the magnetization from $883.31 \mathrm{kA} / \mathrm{m}$ to $955 \mathrm{kA} / \mathrm{m}$ which is still within the tolerance of the magnetization given by the supplier.

From the validated mathematical expressions, the radial force is approximately linear and thus the concept of radial stiffness can be used.

It has been shown that the axial vibrations are best reduced by having a high eddy-current damping directly between rotor and stator. A simple expression by Hahn et al. ${ }^{32}$ for determining the eddy-current damping ratio has been presented and validated for the current application. The expression was found to be in good agreement with experimental results with an average deviation of $9 \%$.

The method is thus useful for determining forces, stiffnesses and axial damping ratio in a fast and efficient way for most relevant configurations of permanent magnet thrust bearings designed for FESS application.

\section{Appendix}

\section{Earnshaw's Theorem and Radial-to-axial Stiffness Ratio}

We consider the potential energy $U$ of a constant magnetic dipole moment $\boldsymbol{\mu}=\left(M_{x}, M_{y}, M_{z}\right)$ in free space, only affected by an external static magnetic field $\mathbf{B}$,

$$
U=-\boldsymbol{\mu} \cdot \mathbf{B}=-M_{x} B_{x}-M_{y} B_{y}-M_{z} B_{z}
$$

with Laplacian

$$
\begin{aligned}
\nabla^{2} U= & -M_{x} \frac{\partial^{2} B_{x}}{\partial x^{2}}-M_{y} \frac{\partial^{2} B_{y}}{\partial x^{2}}-M_{z} \frac{\partial^{2} B_{z}}{\partial x^{2}} \\
& -M_{x} \frac{\partial^{2} B_{x}}{\partial y^{2}}-M_{y} \frac{\partial^{2} B_{y}}{\partial y^{2}}-M_{z} \frac{\partial^{2} B_{z}}{\partial y^{2}} \\
& -M_{x} \frac{\partial^{2} B_{x}}{\partial z^{2}}-M_{y} \frac{\partial^{2} B_{y}}{\partial z^{2}}-M_{z} \frac{\partial^{2} B_{z}}{\partial z^{2}} \\
= & -M_{x} \nabla^{2} B_{x}-M_{y} \nabla^{2} B_{y}-M_{z} \nabla^{2} B_{z} .
\end{aligned}
$$




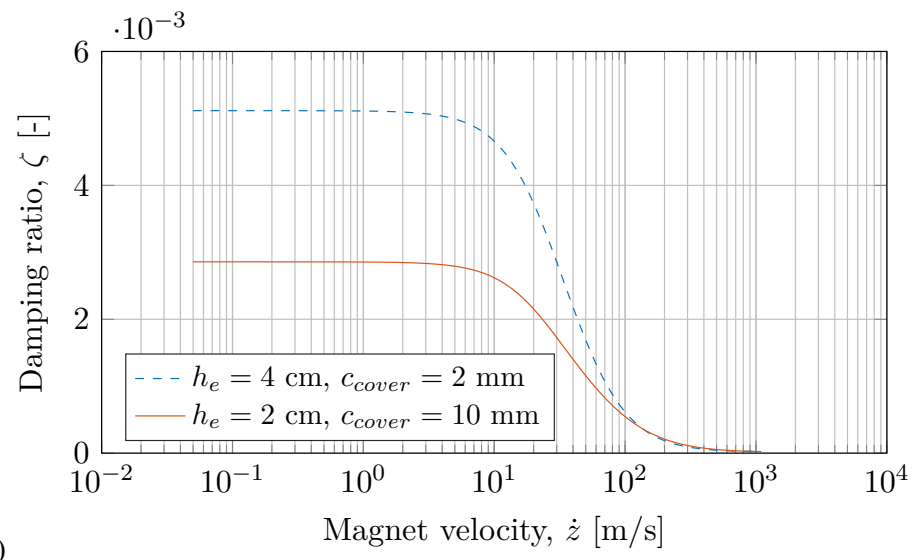

(a)

Figure 13. Damping ratio as a function of the rate of change of magnetic field.

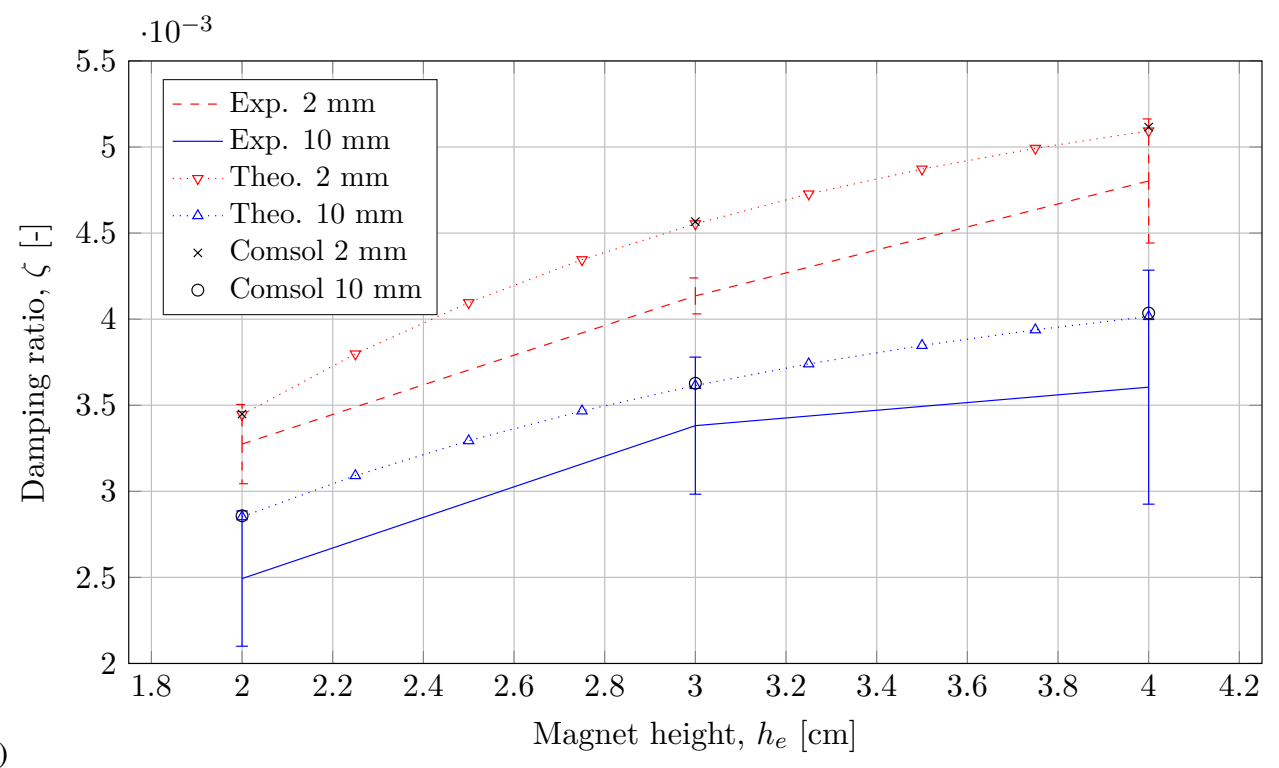

Figure 14. Damping ratio as a function of magnet size and how much of the magnet is covered by the copper tube.

Utilising the identity

$$
\left\{\begin{array}{c}
\nabla^{2} B_{x} \\
\nabla^{2} B_{y} \\
\nabla^{2} B_{z}
\end{array}\right\}=\nabla^{2} \mathbf{B}=\nabla(\nabla \cdot \mathbf{B})-\nabla \times(\nabla \times \mathbf{B})
$$

and the fact that Gauss's and Ampère's law in the absence of any free currents reduces to

$$
\nabla \cdot \mathbf{B}=\mathbf{0} \quad \nabla \times \mathbf{B}=\mathbf{0}
$$

we conclude that

$$
\nabla^{2} U=0
$$

anywhere in free space - also at equilibrium - in accordance with Earnshaw's theorem ${ }^{1}$ (for an equilibrium to be stable, the second derivative of the potential energy must be greater than zero). The conclusion is readily extended to the general case of a collection of dipole moments, as the potential energy from each dipole is additive as long as it is assumed that the magnetic dipole moments are not affecting each other's strength and direction.

Now looking at the dipole fully suspended, in equilibrium, and considering the potential energy of the magnetic spring forces which provide the suspension by means of translational stiffnesses $k_{x}, k_{y}, k_{z}$

$$
U=\frac{1}{2} k_{x} x^{2}+\frac{1}{2} k_{y} y^{2}+\frac{1}{2} k_{z} z^{2}
$$

we obtain

$$
\nabla^{2} U=k_{x}+k_{y}+k_{z}=0
$$

which for the axisymmetric case $k_{x}=k_{y}=k_{r}$, becomes

$$
k_{r}=-\frac{1}{2} k_{z}
$$

\section{Model Parameters}

The model parameters used throughout the article can be seen in Tab. 2-3. 


$\begin{array}{lcccc} & \text { Unit } & \text { Type 1 } & \text { Type 2 } & \text { Type 3 } \\ z_{1}^{\prime}-z_{0}^{\prime} & {[\mathrm{mm}]} & 3 & 6 & 5 \\ r^{\prime} & {[\mathrm{mm}]} & {[202326]} & {[11.520]} & {[813.4]} \\ M_{s} & {[\mathrm{kA} / \mathrm{m}]} & 883-955 & 883-955 & 883-955 \\ \mu_{r} \text { steel } & - & 410 & 410 & 410 \\ n_{z} & & 4 & 4 & 4 \\ n_{\phi} & & 10 & 10 & 10\end{array}$

Table 2. Parameters used in Sec. . $n_{z}$ and $n_{\phi}$ are the number of discretization points used for the numerical integration of Eq. 11.

$\begin{array}{lcc} & \text { Unit } & \\ m_{r} & {[\mathrm{~kg}]} & 3 \\ m_{s} & {[\mathrm{~kg}]} & 0.1 \\ \Omega_{h} & {[\mathrm{~Hz}]} & 0.01-1000 \\ r_{0} & {[\mathrm{~mm}]} & 12.6 \\ r_{1} & {[\mathrm{~mm}]} & 14 \\ \mu_{0} & {[\mathrm{H} / \mathrm{m}]} & 4 \pi \times 10^{-7} \\ n_{z} & & 100 \\ n_{r} & & 5 \\ \omega_{n} & {[\mathrm{~Hz}]} & 15.5 \\ \sigma & {[\mathrm{S} / \mathrm{m}]} & 5.88 \times 10^{7} \\ r_{e} & {[\mathrm{~mm}]} & 9.5 \\ h_{e} & {[\mathrm{~mm}]} & 10-40 \\ z_{\text {cover }} & {[\mathrm{mm}]} & 2 \text { and } 10 \\ M_{s} & {[\mathrm{kA} / \mathrm{m}]} & 883.31\end{array}$

Table 3. Parameters used in Sec. and . $n_{z}$ and $n_{r}$ are the number of discretization points used for the numerical integration of Eq. 21 . The value $M_{s}=883.31 \mathrm{kA} / \mathrm{m}$ is used because it is one of the default magnetizations in FEMM and also within the tolerance of the magnets used for the experiments.

\section{References}

1. Earnshaw S. On the nature of the molecular forces which regulate the constitution of the luminiferous ether., 1839.

2. Wang J. Lift magnet mechanism for flywheel power storage systems: Patent no.: Us7,679,247 b2. US Patent 2010; .

3. Calnetix. Vycon $\AA$ direct connect ( $\operatorname{vdc}()$, 2018. https://www.calnetix.com/ vdc-kinetic-energy-storage-systems [Accessed Feb. 16th 2018].

4. Jansen RH, Dever TP, Jansen RH et al. G2 flywheel module design. Collection of Technical Papers - 2nd International Energy Conversion Engineering Conference 2004; 2: 683-695.

5. Dever T. Presentation: Development of a high specific energy flywheel module, and studies to quantify its mission applications and benefits, 2013. Available online: http://ntrs.nasa.gov/archive/nasa/casi. ntrs.nasa.gov/20150009522.pdf [Accessed Sep. 5th 2016].

6. Lundin J, Kamf T, Abrahamsson J et al. High Speed Flywheels for Vehicular Applications. 14th International Symposium on Magnetic Bearings 2014; : 353-359.

7. Toh CS and Chen SL. Design, modeling and control of magnetic bearings for a ring-type flywheel energy storage system. Energies 2016; 9(12): 1051. DOI:10.3390/en9121051.

8. WattsUp-Power. Flywheel description, 2018. http:// wattsuppower.com/flywheel/ [Accessed Feb. 16th 2018].

9. Zamany $\mathrm{J}$ and Speiermann M. Flywheel for energy storage systems and energy storage systems comprising the same: Wo2016041987a2. Patent 2015; .

10. Veltri JA. Flywheel energy system: Patent no.: Us20110298293a1. US Patent 2010;

11. Yonnet JP. Passive magnetic bearings with permanent magnets. Intermag (int Magn) Conf 1978; MAG-14(5): 803-805.

12. Yonnet JP. Permanent magnet bearings and couplings. IEEE Transactions on Magnetics 1981; MAG-17(1): 1169-1173.

13. Marth E, Jungmayr G, Panholzer $M$ et al. Optimization of Stiffness per Magnet Volume Ratio of Discrete and Continuous Halbach Type Permanent Magnetic Bearings. 13th International Symposium on Magnetic Bearings 2012; : 1-14.

14. Marth E, Jungmayr G, Panholzer M et al. Optimization and realization of a multi-pole permanent magnetic bearing with rotating magnetization. Proceedings of the Institution of Mechanical Engineers Part I-journal of Systems and Control Engineering 2016; 230(4): 320-329. DOI:10.1177/ 0959651815594081.

15. Moser R, Sandtner J and Bleuler H. Optimization of repulsive passive magnetic bearings. IEEE Transactions on Magnetics 2006; 42(8): 2038-2042. DOI:10.1109/TMAG.2005.861160.

16. Lang $M$ and Lembke TA. Design of Permanent Magnet Bearings with high stiffness. Proceedings of ISMB10 2006; $: 1-4$.

17. Bekinal SI and Jana S. Generalized three-dimensional mathematical models for force and stiffness in axially, radially, and perpendicularly magnetized passive magnetic bearings with " $n$ " number of ring pairs. Journal of Tribologytransactions of the ASME 2016; 138(3): 031105. DOI:10.1115/ 1.4032668 .

18. Beneden MVAN, Kluyskens V and Dehez B. Comparison between optimized topologies of permanent magnet thrust bearings with back-iron. Proceedings of ISMB15 2016; (1): 882-888.

19. Van Beneden M, Kluyskens V and Dehez B. Optimal sizing and comparison of permanent magnet thrust bearings. IEEE Transactions on Magnetics 2017; 53(2): 7736118. DOI:10. 1109/TMAG.2016.2625275.

20. Bassani R, Ciulli E, Di Puccio F et al. Study of conic permanent magnet bearings. Meccanica 2001; 36(6): 745-754. DOI: 10.1023/A:1016321525162.

21. Amati N, De Lepine $X$ and Tonoli A. Modeling of electrodynamic bearings. Journal of Vibration and Acousticstransactions of the ASME 2008; 130(6): 061007. DOI:10.1115/ 
1.2981170 .

22. Tonoli A, Amati N, Impinna F et al. A solution for the stabilization of electrodynamic bearings: Modeling and experimental validation. Journal of Vibration and Acousticstransactions of the ASME 2011; 133(2): 021004. DOI:10.1115/ 1.4002959 .

23. Detoni JG. Progress on electrodynamic passive magnetic bearings for rotor levitation. Proceedings of the Institution of Mechanical Engineers, Part C: Journal of Mechanical Engineering Science 2014; 228(10): 1829-1844. DOI:10. 1177/0954406213511798.

24. Filatov AV and Maslen EH. Passive magnetic bearing for flywheel energy storage systems. IEEE Transactions on Magnetics 2001; 37(6): 3913-3924. DOI:10.1109/20.966127.

25. Sandtner $\mathbf{J}$ and Bleuler H. Compact Passive Electrodynamic Thrust Bearing- Electrodynamic Bearings-. 11th International Symposium on Magnetic Bearings 2008; : 347-350.

26. Sandtner J and Bleuler H. Passive Electrodynamic Magnetic Thrust Bearing Especially Designed for Constant Speed Applications. 10th International Symposium on Magnetic Bearings 2006; .

27. Siebert M, Ebihara B, Jansen R et al. A passive magnetic bearing flywheel. 36th Intersociety Enegy Conversion Engineering Conference 2002; .

28. Morales W, Fusaro R and Kascak A. Permanent magnetic bearing for spacecraft applications. Tribology Transactions 2003; 46(3): 460-464.

29. Bekinal SI, Kulkarni SS and Jana S. A hybrid (permanent magnet and foil) bearing set for complete passive levitation of high-speed rotors. Proceedings of the Institution of Mechanical Engineers Part C-journal of Mechanical Engineering Science 2017; 231(20): 3679-3689. DOI:10.1177/0954406216652647.

30. Fremerey JK and Kolk M. A $500-$ Wh power flywheel on permanent magnet bearings. Proceedings of ISMST5 ; : 287295.

31. Lembke T. 1-Dof Bearing Arrangement with Passive Radial Bearings and Highly Efficient Integrated Electrodynamic Dampers, EDD. 13th International Symposium on Magnetic Bearings 2012; : 1-7.

32. Hahn K, Johnson E, Brokken A et al. Eddy current damping of a magnet moving through a pipe. American Journal of Physics 1998; 66(12): 1066-1076. DOI:10.1119/1.19060.

33. Hedlund M, Abrahamsson J, Perez-Loya JJ et al. Eddy currents in a passive magnetic axial thrust bearing for a flywheel energy storage system. International Journal of Applied Electromagnetics and Mechanics 2017; 54(3): 389-404. DOI: 10.3233/JAE-160151.

34. Berry MV. The levitron: an adiabatic trap for spins. Proceedings of the Royal Society of London, Series a (mathematical, Physical and Engineering Sciences) 1996; 452(1948): 1207-20, 1207-1220. DOI:10.1098/rspa.1996. 0062.

35. Genta G, Delprete C and Rondano D. Gyroscopic stabilization of passive magnetic levitation. Meccanica 1999; 34(6): 411424. DOI:10.1023/A:1004704428634.

36. Gasch R and Lang M. Levitron - ein beispiel für die rein permanentmagnetische lagerung eines rotors. Zamm Zeitschrift Fur Angewandte Mathematik Und Mechanik 2000; 80(2): 137-144. DOI:10.1002/(SICI)1521-4001(200002)80:2〈137:: AID-ZAMM137>3.0.CO;2-X.

37. Fujii N. Magnet wheel using permanent magnets for repulsive magnetic levitation and induction type thrust. Proceedings of ISMST4 1997; : 165-178.

38. Simon M, Heflinger L and Ridgway S. Spin stabilized magnetic levitation. American Journal of Physics 1997; 65(4): 286-292. DOI:10.1119/1.18488.

39. Conway J. Exact solutions for the magnetic fields of axisymmetric solenoids and current distributions. IEEE Transactions on Magnetics 2001; 37(4): 2977-2988. DOI: 10.1109/20.947050.

40. Snow. Magnetic field of cylindrical and annular coils. Applied Mathematics Series, National Bureau of Standards 1953; (38): 29 pp., 29 pp.

41. Kolbenheyer T. Das magnetische feld eines geraden kreiszylinders bei homogener magnetisierung. Studia Geophysica Et Geodaetica 1964; 8(2): 162-173.

42. Varga E and Beyer A. Magnetic field of a uniformly magnetized hollow cylinder. IEEE Transactions on Magnetics 1998; 34(3): 613-618. DOI:10.1109/20.668053.

43. Urankar LK. Vector potential and magnetic field of currentcarrying finite arc segment in analytical form. ii. thin sheet approximation. IEEE Transactions on Magnetics 1982; MAG18(3): 911-17, 911-917. DOI:10.1109/TMAG.1982.1061927.

44. Lang M. Fast calculation method for the forces and stiffnesses of permanent-magnet bearings. 8th International Symposium on Magnetic Bearing 2002; : 533-538.

45. Meeker D. Web page: Finite element method magnetics, 2013. Available online: http: //www. femm. info/wiki/ HomePage [Accessed Apr. 23rd 2018].

46. COMSOL. Magnetic stiffness of an axial magnetic bearing in 3d, application id: 27761, 2017. Available online: https://www.comsol.com/model/ magnetic-stiffness-of-an-axial-magnetic-bearing-in [Accessed May. 25th 2018].

47. Detoni JG, Cui Q, Amati $\mathrm{N}$ et al. Modeling and evaluation of damping coefficient of eddy current dampers in rotordynamic applications. Journal of Sound and Vibration 2016; 373: 5265. DOI:10.1016/j.jsv.2016.03.013. 